\title{
REVISIONES TEMÁTICAS
}

\section{ENDOSCOPIA AVANZADA: ¿CAFE PARA TODOS?}

\section{ADVANCED ENDOSCOPY: IS IT FOR EVERYONE?}

\section{Rodríguez Ramos C}

UCG de Aparato Digestivo. Hospital Universitario Puerta del Mar. Cádiz. Servicio de Aparato Digestivo.

Hospital Quironsalud Campo de Gibraltar. Los Barrios, Cádiz.

\section{Resumen}

Como consecuencia del avance tecnológico experimentado durante las últimas décadas en la endoscopia digestiva se ha incrementado notablemente la complejidad en esta disciplina, tanto con el desarrollo de nuevas técnicas como con la ampliación del campo de acción de técnicas ya establecidas.

La implantación de técnicas de endoscopia avanzada requiere una adecuada formación previa y una cuidadosa planificación de las condiciones en que se va a desarrollar dicha actividad con el objeto de conseguir para nuestros pacientes unos resultados adecuados en seguridad, eficacia y eficiencia de los procedimientos realizados.

En este artículo de controversia planteamos una serie de consideraciones acerca de la forma en que aprendemos, enseñamos y realizamos técnicas de endoscopia avanzada en nuestro medio, con el objetivo de promover el debate sobre su idoneidad y posibles alternativas, si las hubiera.

\section{CORRESPONDENCIA}

Claudio Rodriguez Ramos

Hospital Universitario Puerta del Mar

11009 Cádiz.

claudioa.rodriguez.sspa@juntadeandalucia.es

Fecha de envío: 27/12/2019

Fecha de aceptación: 10/01/2020
Palabras clave: endoscopia digestiva avanzada, aprendizaje, competencia, habilidades.

\section{Abstract}

As a consequence of the technological progress experienced during the last decades in digestive endoscopy, the complexity in this discipline has increased significantly, both with the development of new procedures and with the extension of the field of action of already established techniques.

The implementation of advanced endoscopy techniques requires adequate prior training and careful planning of the conditions under which this activity will be carried out in order to achieve optimal results for safety, efficacy and efficiency of the procedures performed.

In this controversy article we raise a number of considerations about how we learn, teach and perform advanced endoscopy techniques in our environment, with the aim of promoting the debate about their suitability and possible alternatives, if any.

Keywords: advanced gastrointestinal endoscopy, training, competence, skills. 


\section{Introducción. Concepto de endoscopia avanzada.}

El término endoscopia avanzada se utiliza para hacer referencia a aquellos procedimientos endoscópicos de mayor dificultad intrínseca y que exigen la posesión de un nivel más elevado de habilidades endoscópicas. Suelen requerir además un equipamiento y utillaje diferenciado y precisan un aprendizaje específico, generalmente tutelado, que suele adquirirse una vez finalizado el periodo de formación reglada de la especialidad. Incluye, entre otros procedimientos, la colangiopancreatografía retrógrada endoscópica (CPRE), la ecoendoscopia, la colocación de prótesis digestivas, la enteroscopia, la resección mucosa endoscópica (REM) de lesiones complejas, la disección submucosa endoscópica (DES), la miotomía peroral endoscópica (POEM) o la endoscopia bariátrica.

Es cierto que, en un sentido amplio, toda innovación técnica o tecnológica en la endoscopia digestiva se denomina en ocasiones endoscopia avanzada. Sin embargo, es preferible no incluir en este concepto aquellos avances que son la extensión de técnicas ampliamente disponibles (colocación de sobreclips, aplicación de adhesivos, gastrostomía con gastropexia, resección mucosa de lesiones pequeñas, etc) y no suponen la adquisición de habilidades diferenciadas.

La realización de estas técnicas mediante la incorporación a un equipo de endoscopia avanzada ya consolidado o mediante la inclusión de procedimientos de endoscopia avanzada a la cartera de servicios de nuestros centros resulta un proyecto sumamente atractivo para cualquier endoscopista vocacional por dos razones fundamentales: la primera es el beneficio que pueden aportar a la atención de nuestros pacientes ya que estas técnicas permiten abordar problemas clínicos que quedan fuera del alcance de las técnicas básicas $y$, concretamente en su vertiente terapéutica, constituyen una alternativa menos agresiva frente a la opción de tratamiento quirúrgico de múltiples patologías; la segunda es que la realización de estos procedimientos avanzados es percibida como la forma natural de progresión profesional en el campo de la endoscopia.

Sin embargo, los procedimientos avanzados, en comparación con los procedimientos endoscópicos básicos, presentan un mayor grado de dependencia del operador, acumulan un mayor porcentaje de complicaciones graves, requieren un mayor tiempo medio de ejecución y suponen un mayor coste. Todo ello plantea importantes dificultades para conseguir una adecuada formación en estas técnicas y para situarlas correctamente en la práctica clínica en términos de efectividad, eficiencia y de seguridad para el paciente, tanto en términos absolutos como frente a otras técnicas ya establecidas.

En nuestro país la obtención de la competencia para la realización de endoscopia básica se realiza, o debiera realizarse, durante el periodo de formación en la especialidad de Aparato Digestivo. Sin embargo, la formación en endoscopia avanzada no está regulada actualmente y se encuentra en espera del desarrollo de un área de capacitación específica ${ }^{1,2}$. Por ello, la adquisición de la competencia en procedimientos avanzados debe realizarse inevitablemente tras la residencia, generalmente de forma simultánea al ejercicio profesional como especialista y mediante "itinerarios formativos autodiseñados" por el propio endoscopista en los que se incluyen el estudio de bibliografía específica, la asistencia a cursos teórico-prácticos y a demostraciones en directo, la visita o la estancia en unidades especializadas, etc. Aunque es obligado reconocer el esfuerzo y la dedicación que debe realizar el endoscopista que decide encaminar su trayectoria profesional por este camino debe admitirse igualmente que es cada vez más necesario disponer de itinerarios formativos diseñados y avalados por las administraciones competentes y/o por las sociedades científicas que permitan asegurar y certificar la consecución de un nivel adecuado de competencia.

\section{Formación en endoscopia avanzada: adquisición y valoración de la competencia.}

\section{Habilidades endoscópicas}

La formación en endoscopia avanzada tiene como finalidad adquirir la competencia necesaria para realizar procedimientos endoscópicos avanzados de forma independiente.

En este contexto entendemos la competencia en endoscopia como el nivel mínimo de habilidad, conocimiento y/o pericia, adquirido mediante el aprendizaje y la experiencia, que se requiere para realizar de forma segura y cualificada un procedimiento endoscópico. Se basa en la posesión de un conjunto de habilidades psicomotoras, cognitivas e integradoras ${ }^{3}$.

Las habilidades psicomotoras (fundamentalmente del ámbito de la motricidad fina y de la coordinación óculomotora) tienen un considerable componente innato, pero pueden perfeccionarse mediante el aprendizaje y la práctica. En este grupo se incluyen el manejo de los mandos y de la torsión del endoscopio, la manipulación del instrumental (asas, guías, agujas de punción...), la inspección de la mucosa, etc.

Las competencias cognitivas se refieren al conocimiento de los fundamentos anatómicos, clínicos y técnicos de los procedimientos endoscópicos, así como a la aplicación de la información obtenida endoscópicamente a la práctica clínica: indicaciones, contraindicaciones y complicaciones de cada procedimiento, identificación de lesiones, características técnicas del equipamiento, manejo de fármacos sedantes, etc.

Las habilidades psicomotoras y las competencias cognitivas son necesarias, pero no suficientes, para asegurar la competencia en endoscopia: precisan de las denominadas competencias integradoras. Éstas son habilidades no técnicas que complementan a las anteriores y permiten la dispensación al paciente de una endoscopia de calidad, influyendo decisivamente en la seguridad y en el resultado del procedimiento. A este grupo pertenecen competencias como la actitud ante el riesgo, la capacidad de toma de decisiones, la capacidad de trabajo en equipo, la tolerancia a la incertidumbre, la capacidad de contextualizar los hallazgos endoscópicos etc. ${ }^{3,4}$.

\section{Herramientas e itinerarios formativos}

En espera del desarrollo a nivel nacional de programas oficiales de formación en endoscopia avanzada, a nivel europeo disponemos de algunas recomendaciones acerca de la formación en 
endoscopia avanzada que pueden ser útiles para indicarnos como puede realizarse la adquisición de habilidades en este ámbito.

En el Blue Book de 2017, editado por The European Section and Board of Gastroenterology \& Hepatology, se indica que el periodo de subespecialización en endoscopia avanzada debería realizarse durante uno o dos años en unidades con un volumen mínimo de casos anuales para cada uno de los procedimientos que se incluyen en el curriculum ${ }^{5}$. Sin embargo, este sistema de aprendizaje fundamentado en la realización de un número mínimo de casos durante un tiempo determinado y con una transmisión de conocimientos basada en el modelo maestro-aprendiz se considera inadecuado hoy en día para garantizar la adquisición de competencia en endoscopia avanzada ya que se ha demostrado que existe una amplia variabilidad interindividual en las curvas de aprendizaje de estos procedimientos debido a diferencias en la posesión de habilidades innatas, en la experiencia previa o en la calidad del entrenamiento ${ }^{3}$.

Por ello resultan más apropiadas las indicaciones de la European Society of Gastrointestinal Endoscopy (ESGE) en su proyecto de elaboración de un curriculum de formación postgraduada para distintos procedimientos de endoscopia avanzada $^{6}$, en el que se propone un sistema de aprendizaje basado en la utilización de herramientas de evaluación de habilidades para valorar los resultados de la formación y confirmar la adquisición de una adecuada competencia ${ }^{7,8}$.

Este tipo de herramientas estructuradas están disponibles actualmente para procedimientos básicos (gastroscopia y colonoscopia) $)^{9,10}$, para CPRE y para ecoendoscopia ${ }^{7,11}$. Para la valoración de competencias en disección submucosa endoscópica en el mundo occidental podemos encontrar paneles de recomendaciones que ha sido elaborados mediante conferencias de consenso ${ }^{12}$. Además, se han publicado de numerosos indicadores no agrupados para la valoración del desempeño del endoscopista durante la formación en endoscopia avanzada ${ }^{13}$.

No es el objeto de este artículo la exposición detallada de estos paneles de evaluación, pero debemos señalar la importancia, a la hora de organizar un proceso de formación en endoscopia avanzada, de disponer de herramientas de evaluación y de indicadores que, por una parte, nos indican qué habilidades son más trascendentes para obtener la competencia adecuada y en las que, por tanto, debemos poner especial empeño para su aprendizaje; y por otra. nos permiten determinar la consecución de un nivel adecuado de competencia de forma más fiable que mediante la realización de un número determinado de casos.

Una vez identificadas las habilidades precisas a conseguir, el siguiente paso es el diseño del itinerario formativo. La formación en endoscopia avanzada debe realizarse mediante la superación de etapas escalonadas, de dificultad progresiva, que permitan la adquisición de las habilidades con una secuencia lógica (Figura 1). Estas etapas pueden ordenarse de forma genérica de la siguiente forma ${ }^{6,14}$ :

1. Asegurar la posesión de habilidades técnicas y cognitivas previas necesarias para iniciar la formación en un procedimiento avanzado específico, con especial atención a la capacidad de
ITINERARIO FORMATIVO GENÉRICO PARA ENDOSCOPIA AVANZADA
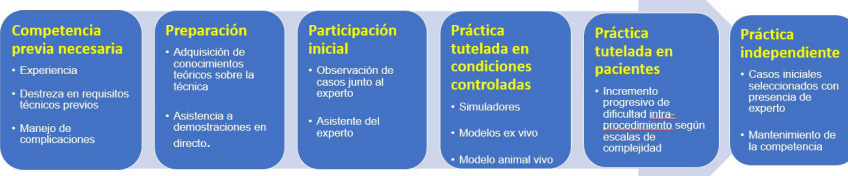

Estructura formal:

- Registro

- Supervisión externa

- Evaluación objetiva

Figura 1

Itinerario formativo genérico para endoscopia avanzada.

resolver complicaciones que pueden presentarse durante los procedimientos endoscópicos: hemorragia, perforación, deterioro súbito del paciente, etc.

2. Preparación para la formación: adquisición de conocimientos teóricos sobre la técnica, asistencia a demostraciones en directo.

3. Participación inicial en el procedimiento: observación de casos junto al experto, asistencia al experto durante la realización de la técnica.

4. Realización de la técnica en situación controlada: simuladores, modelos ex vivo, modelos animales vivos.

5. Realización de casos en paciente con tutorización. Esta fase queda lógicamente supeditada a la normativa que regule la posibilidad de realización de actos médicos sobre pacientes a los endoscopistas que no formen parte de la plantilla del centro.

6. Incremento progresivo de dificultad intraprocedimiento según escalas de complejidad ${ }^{15,16}$

7. Práctica independiente. Mantenimiento de la competencia.

Para culminar el proceso de formación debería existir una supervisión externa cualificada que pudiera certificar la adquisición de un grado suficiente de competencia en el procedimiento. En ausencia de dicha figura, como ocurre en nuestro medio, únicamente podrán realizarse valoraciones parciales: valoraciones por el equipo docente o una autovaloración, con las limitaciones que ello conlleva. Es importante poseer un registro de las actividades realizadas y los objetivos alcanzados en cada una de ellas con respecto a las habilidades a evaluar para facilitar dicha evaluación.

\section{Inicio de la actividad independiente en edoscopia avanzada}

Una vez concluida la etapa de formación y antes de iniciar la actividad independiente es recomendable valorar los inconvenientes que acompañan a la práctica de la endoscopia avanzada y nuestra capacidad para afrontarlos. El porcentaje de complicaciones y la gravedad de éstas es mayor que en los procedimientos convencionales, 
como ya hemos indicado, y si esta situación no es adecuadamente manejada puede originar un elevado nivel de estrés ${ }^{17-19}$. Por otra parte, en nuestro medio tampoco existe una motivación económica que justifique por si misma la elección de la endoscopia avanzada como actividad profesional. En el sistema sanitario público no existe una remuneración diferenciada para la realización de estos procedimientos y en la actividad privada los baremos no suelen superar proporcionalmente a los que poseen procedimientos de endoscopia convencional, de tal forma que la realización de un pequeño número de procedimientos convencionales puede igualar el importe adjudicado a procedimientos avanzados.

Otro aspecto importante es que, en función del procedimiento que queramos introducir en la práctica clínica de nuestro centro, debemos revisar previamente si existen recomendaciones de agencias gubernamentales (p. ej. agencias de evaluación de tecnologías sanitarias) acerca de su realización que nos indiquen las condiciones en que debemos implantarlo ${ }^{20-23}$.

El inicio de la actividad independiente es un momento crítico en la implantación de técnicas de endoscopia avanzada ya que de los resultados iniciales dependerá en buena medida la consolidación de la técnica (11.12,14,24. Por todo ello, los principales aspectos que se deben programar para comenzar a realizar un determinado procedimiento serían:

1. Asegurar una formación adecuada del endoscopista, a ser posible certificada de forma externa.

2. Cumplir los requisitos administrativos preceptivos: conformidad de la Dirección de la unidad, de la Dirección del centro, de la Junta facultativa y del Comité de ética.

3. Disponer en el hospital de los servicios de apoyo necesarios para garantizar un resultado adecuado y minimizar el daño ocasionado por posibles complicaciones (cirugía, anestesia, $\mathrm{UCl}$, anatomía patológica, radiología, etc.). Estos servicios deben estar informados y dar su conformidad.

4. Planificar los aspectos organizativos y logísticos: disponibilidad de material adecuado, personal auxiliar formado, organización de agendas para disponer de tiempo suficiente, acondicionamiento de la sala, programación de ingreso hospitalario y seguimiento posterior etc.

5. Recoger los datos de los procedimientos en un registro prospectivo, individual o colectivo, de recogida de información acerca de los resultados de la técnica desde el inicio del programa, de cara a asegurar su correcto funcionamiento y la ausencia de desviaciones inesperadas respecto a los estándares $21,25,26$.

6. Evaluar la actividad realizada mediante herramientas de valoración de la competencia ${ }^{11}$.

Por otra parte, es fundamental seleccionar cuidadosamente los casos a realizar en esta primera fase de la implantación de una técnica de endoscopia avanzada. Los principales criterios serían:
1. Atenerse a las indicaciones plenamente aceptadas para la técnica.

2. Elegir los casos de menor dificultad técnica previsible.

3. Informar adecuadamente al paciente, comprobando su comprensión acerca de la técnica a realizar y de su reciente introducción en el Centro.

Por último, es sumamente deseable la presencia de un experto en los casos iniciales, no tanto para tutorizar al endoscopista como para prestar apoyo en caso necesario. Esta no es una condición fácilmente alcanzable pero con el desarrollo de herramientas como la tecnología $5 \mathrm{G}$ y la realidad aumentada para integración de datos médicos puede ser posible disponer de teleasistencia por parte de endoscopistas expertos, como quedó demostrado en un reciente curso realizado en Málaga ${ }^{17}$.

\section{Consolidación de la actividad independiente en endoscopia avanzada. Mantenimiento de la competencia y cumplimiento de estándares de calidad.}

Para asegurar el mantenimiento de la competencia en endoscopia avanzada es de capital importancia disponer de unvolumen adecuado de casos ya que bajos volúmenes de procedimientos se correlacionan con un mayor número de complicaciones ${ }^{28-31}$. Las cifras recomendadas varían según el procedimiento ${ }^{14,30}$, pero de nuevo se debe hacer hincapié en el empleo adicional de indicadores de calidad del procedimiento referidas a resultados y complicaciones para asegurar una adecuada utilización y ejecución de la técnica ${ }^{11,14,32-34}$.

En esta fase de la práctica de procedimientos avanzados es importante no relajar la adecuación en la indicación de la técnica con el objeto de mantener un número adecuado de procedimientos ya que puede comprometerse la seguridad, la eficacia y la eficiencia del programa ${ }^{35,36}$. En ocasiones puede incluso considerarse la centralización del procedimiento al objeto de asegurar la adecuada realización del mismo $^{37}$.

Para todas estas decisiones la comparación de indicadores entre centros y endoscopistas (benchmarking) sería una herramienta de gran utilidad, aunque su implantación puede ser compleja y requiere de una intensa cooperación entre los distintos actores implicados ${ }^{36,38}$

\section{Progresión de la actividad independiente en endoscopia avanzada. Aumento de la complejidad. Actividad docente.}

La evolución natural de un programa de endoscopia avanzada es la asunción progresiva de casos de mayor complejidad, para lo cual es importante mantener los estándares de volumen y resultados. Pueden servirnos de orientación para ello diversas guías de complejidad de procedimientos y escalas de predicción de dificultad en el abordaje de lesiones 12,16,39-42. $^{\text {. }}$ 
Por último, siempre que nos lo permita la actividad asistencial, es deseable plantear el desarrollo de actividades docentes que faciliten la correcta difusión de la endoscopia avanzada a la vez que contribuyen al perfeccionamiento de los propios procedimientos y al mantenimiento de la motivación necesaria para la persistencia de nuestro programa. Para ello es importante adquirir habilidades docentes específicas a través de fuente bibliográficas ${ }^{43,44}$ y/o mediante la participación en actividades específicas ${ }^{45}$.

\section{Bibliografía}

1. Orden SAS/2854/2009, de 9 de octubre, por la que se aprueba y publica el programa formativo de la especialidad de Aparato Digestivo. BOE-A-2009-16993.

2. Brotons Á, Vilella A, Sánchez-Montes C, Garau C, Vila A, Pons Beltrán V et al. Formación básica en endoscopia digestiva para los médicos residentes de la especialidad de Aparato Digestivo. Recomendaciones de la Sociedad Española de Endoscopia Digestiva (SEED). Rev Esp Enferm Dig. 2019;111(3):228-38.

3. Walsh CM. In-training gastrointestinal endoscopy competency assessment tools: Types of tools, validation and impact. Best Pract Res Clin Gastroenterol. 2016 Jun;30(3):357-74.

4. Hitchins CR, Metzner M, Edworthy J, Ward C. Non-technical skills and gastrointestinal endoscopy: a review of the literature. Frontline Gastroenterol. 2018 Apr;9(2):129-34.

5. The European Section and Board of Gastroenterology and Hepatlogy. Higher training modules. Interventional endoscopy curriculum. In: The ESBGH Training Programme The Blue Book. 2017. p. 40-1.

6. Bisschops R, Dekker E, East JE, Johnson G, Pimentel-Nunes P, Sanders DS, et al. European Society of Gastrointestinal Endoscopy (ESGE) curricula development for postgraduate training in advanced endoscopic procedures: rationale and methodology. Endoscopy. 2019 Oct;51(10):976-9.

7. Siau K, Dunckley P, Feeney M, Johnson G. ERCP assessment tool: evidence of validity and competency development during training. Endoscopy. 2019 Nov;51(11):1017-26.

8. Wani S, Keswani RN, Petersen B, Edmundowicz SA, Walsh CM, Huang C, et al. Training in EUS and ERCP: standardizing methods to assess competence. Gastrointest Endosc. 2018 Jun;87(6):1371-82.

9. Siau K, Crossley J, Dunckley P, Johnson G, Feeney M, lacucci M, et al. Colonoscopy Direct Observation of Procedural Skills Assessment Tool for Evaluating Competency Development During Training. Am J Gastroenterol. 2019 Nov; doi: 10.14309/ajg.0000000000000426. [Epub ahead of print]

10. Siau K, Crossley J, Dunckley P, Johnson G, Feeney M, Hawkes ND, et al. Direct observation of procedural skills (DOPS) assessment in diagnostic gastroscopy: nationwide evidence of validity and competency development during training. Surg Endosc. 2019 Mar; doi: 10.1007/s00464-019-06737-7. [Epub ahead of print]

11. Wani S, Keswani RN, Han S, Aagaard EM, Hall M, Simon V, et al. Competence in Endoscopic Ultrasound and Endoscopic Retrograde Cholangiopancreatography, From Training Through Independent Practice.
Gastroenterology. 2018 Nov;155(5):1483-1494.e7.

12. Oyama T, Yahagi N, Ponchon T, Kiesslich T, Berr F. How to establish endoscopic submucosal dissection in Western countries. World J Gastroenterol. 2015 Oct;21(40):11209-20.

13. James PD, Antonova L, Martel M, Barkun A. Measures of trainee performance in advanced endoscopy: A systematic review. Best Pract Res Clin Gastroenterol. 2016 Jun;30(3):421-52.

14. Pimentel-Nunes $P$, Pioche $M$, Albeniz E, Berr F, Deprez P, Ebigbo A, et al. Curriculum for endoscopic submucosal dissection training in Europe: European Society of Gastrointestinal Endoscopy (ESGE) Position Statement. Endoscopy. 2019 Oct;51(10):980-92.

15. Jorgensen J, Kubiliun N, Law JK, Al-Haddad MA, Bingener-Casey J, Christie $J A$, et al. Endoscopic retrograde cholangiopancreatography (ERCP): core curriculum. Gastrointest Endosc. 2016 Feb;83(2):279-89.

16. Olsson G, ArneloU, SwahnF, TornqvistB, Lundell L, Enochsson L.TheH.O.U.S.E. classification: a novel endoscopic retrograde cholangiopancreatography (ERCP) complexity grading scale. BMC Gastroenterol. 2017 Mar;17(1):38.

17. Koo P, Yung V, Dutta A, Sarkar S. Choosing a Career in Advanced Endoscopy or General Gastroenterology. Dig Dis Sci. 2017 Jun;62(6):1409-11.

18. Gleeson D, O'Shea C, Ellison H, Tham TC, Douds AC, Goddard AF. Stress and its causes in UK gastroenterologists: results of a national survey by the British Society of Gastroenterology. Frontline Gastroenterol. 2019 Jan;10(1):43-9.

19. Keswani RN, Taft TH, Cote GA, Keefer L. Increased levels of stress and burnout are related to decreased physician experience and to interventional gastroenterology career choice: findings from a US survey of endoscopists. Am J Gastroenterol. 2011 Oct;106(10):1734-40.

20. Haute Autorité de santé. Traitement endoscopique par dissection sousmuqueuse des lésions cancéreuses superficielles coliques Rapport d'évaluation technologique [Internet]. 2019. Available from: https://www.has-sante.fr/ upload/docs/application/pdf/2019-02/rapport_dsm_colon_vd.pdf

21. Benguria-Arrate G, Gutiérrez-Ibarluzea I GL. Resección Mucosa Endoscópica (RME) y Disección Submucosa Endoscópica (DSE) en cánce-res gastrointestinales superficiales. [Internet]. Ministerio de Sanidad, Servicios Sociales e Igualdad. Servicio de Evaluación de Tecnologías Sanitarias del País Vasco; 2016. Available from: http://www.ogasun.ejgv.euskadi.eus/r51-catpub/ es/k75aWebPublicacionesWar/k75aObtenerPublicacionDigitalServlet?R01HN oPortal=true\&N_LIBR=051939\&N_EDIC $=0001 \& C$ IDIOM $=e s \& F O R M A T O=. p d f$

22. Haute Autorité de santé. Traitement endoscopique par dissection sousmuqueuse des cancers superficiels de l'estomac Rapport d'évaluation technologique [Internet]. Available from: https://www.has-sante.fr/upload/ docs/application/pdf/2018-12/rapport_dsm_estomac_vd.pdf

23. Haute Autorité de santé. Traitement endoscopique par dissection sousmuqueuse des cancers superficiels de l'oesophage Rapport d'évaluation technologique [Internet]. Available from: https://www.has-sante.fr/upload/ docs/application/pdf/2018-12/rapport_dsm_oesophage_vd.pdf

24. Faulx AL, Lightdale JR, Acosta RD, Agrawal D, Bruining DH, Chandrasekhara $\mathrm{V}$, et al. Guidelines for privileging, credentialing, and proctoring to perform $\mathrm{GI}$ 
endoscopy. Gastrointest Endosc. 2017 Feb;85(2):273-81.

25. Lee TJ, Siau K, Esmaily S, Docherty J, Stebbing J, Brookes MJ, et al. Development of a national automated endoscopy database: The United Kingdom National Endoscopy Database (NED). United Eur Gastroenterol J. 2019 Jul;7(6):798-806.

26. https://www.wseed.org/index.php/quienes-somos/grupos-de-trabajo [Internet]. Available from: https://www.wseed.org/index.php/quienes-somos/ grupos-de-trabajo

27. Serrano R. Realidad aumentada y tecnología $5 \mathrm{G}$ revolucionan la endoscopia digestiva. DIARIO MÉDICO [Internet]. 2019 Oct 10; Available from: https:// www.diariomedico.com/tecnologia/realidad-aumentada-y-tecnologia-5grevolucionan-la-endoscopia-digestiva.html

28. Varadarajulu S, Kilgore ML, Wilcox CM, Eloubeidi MA. Relationship among hospital ERCP volume, length of stay, and technical outcomes. Gastrointest Endosc. 2006 Sep;64(3):338-47.

29. Markar SR, Mackenzie H, Ni M, Huddy JR, Askari A, Faiz O, et al. The influence of procedural volume and proficiency gain on mortality from upper Gl endoscopic mucosal resection. Gut. 2018 Jan;67(1):79-85.

30. Keswani RN, Qumseya BJ, O’Dwyer LC, Wani S. Association Between Endoscopist and Center Endoscopic Retrograde Cholangiopancreatography Volume With Procedure Success and Adverse Outcomes: A Systematic Review and Meta-analysis. Clin Gastroenterol Hepatol. 2017 Dec;15(12):1866-1875. e3.

31. Huang RJ, Barakat MT, Girotra M, Lee JS, Banerjee S. Unplanned Hospital Encounters After Endoscopic Retrograde Cholangiopancreatography in 3 Large North American States. Gastroenterology. 2019 Jan;156(1):119-129.e3.

32. Domagk D, Oppong KW, Aabakken L, Czako L, Gyokeres T, Manes G, et al. Performance measures for endoscopic retrograde cholangiopancreatography and endoscopic ultrasound: A European Society of Gastrointestinal Endoscopy (ESGE) Quality Improvement Initiative. United Eur Gastroenterol J. 2018 Dec;6(10):1448-60.

33. Fuccio L, Bhandari P, Maselli R, Frazzoni L, Ponchon T, Bazzoli F, et al. Ten quality indicators for endoscopic submucosal dissection: what should be monitored and reported to improve quality. Ann Transl Med. 2018 Jul;6(13):262.

34. Alberca de Las Parras F, Lopez-Picazo J, Perez Romero S, Sanchez Del Rio A, Judez Gutierrez J, Leon Molina J. Quality indicators for endoscopic retrograde cholangiopancreatography. The procedure of endoscopic retrograde cholangiopancreatography. Rev Esp Enferm Dig. 2018 Oct;110(10):658-66.

35. Kim TJ, Kim ER, Hong SN, Kim Y-H, Chang DK. Current practices in endoscopic submucosal dissection for colorectal neoplasms: a survey of indications among Korean endoscopists. Intest Res. 2017 Apr;15(2):228-35.

36. Katzarov AK, Dunkov ZI, Popadiin I, Katzarov KS. How to measure quality in endoscopic retrograde cholangiopancreatography (ERCP). Ann TransI Med. 2018 Jul;6(13):265.

37. Muller AF. Endoscopic retrograde cholangiopancreatography: is the centre better? The case against centralisation of ERCP services. Frontline Gastroenterol [Internet]. 2013 Jul 1;4(3):210 LP - 212. Available from: http:// fg.bmj.com/content/4/3/210.abstract

38. Kapral C, Muhlberger A, Wewalka F, Duller C, Knoflach P, Schreiber F. Quality assessment of endoscopic retrograde cholangiopancreatography: results of a running nationwide Austrian benchmarking project after 5 years of implementation. Eur J Gastroenterol Hepatol. 2012 Dec;24(12):1447-54.

39. Lakhtakia S. Complications of diagnostic and therapeutic Endoscopic Ultrasound. Best Pract Res Clin Gastroenterol. 2016 Oct;30(5):807-23.

40. Fabbri C, Luigiano C, Lisotti A, Cennamo V, Virgilio C, Caletti G, et al. Endoscopic ultrasound-guided treatments: are we getting evidence based--a systematic review. World J Gastroenterol. 2014 Jul;20(26):8424-48.

41. Iacopini F, Saito Y, Bella A, Gotoda T, Rigato P, Elisei W, et al. Colorectal endoscopic submucosal dissection: predictors and neoplasm-related gradients of difficulty. Endosc Int open. 2017 Sep;5(9):E839-46.

42. Sidhu M, Tate DJ, Desomer L, Brown G, Hourigan LF, Lee EYT, et al. The size, morphology, site, and access score predicts critical outcomes of endoscopic mucosal resection in the colon. Endoscopy. 2018 Jul;50(7):684-92.

43. Wyles SM, Schwarz E, Dort J, Tariq N, Cecil T, Coleman MG, et al. SAGE(S) advice: application of a standardized train the trainer model for faculty involved in a Society of American Gastrointestinal and Endoscopic Surgeons (SAGES) hands-on course. Surg Endosc. 2017 May;31(5):2017-22.

44. Waschke KA, Anderson J, Macintosh D, Valori RM. Training the gastrointestinal endoscopy trainer. Best Pract Res Clin Gastroenterol. 2016 Jun;30(3):409-19.

45. World Gastroenterology Organisation. Train the trainers [Internet]. Available from: https://www.worldgastroenterology.org/education-andtraining/train-the-trainers. 